\title{
A CHARACTERIZATION OF WEIGHTED BERGMAN-ORLICZ SPACES ON THE UNIT BALL IN $\mathbb{C}^{n}$
}

\author{
YASUO MATSUGU and JUN MIYAZAWA
}

(Received 6 April 2001; revised 8 October 2001)

Communicated by P. C. Fenton

\begin{abstract}
Let $B$ denote the unit ball in $\mathbb{C}^{n}$, and $v$ the normalized Lebesgue measure on $B$. For $\alpha>-1$, define $d v_{\alpha}(z)=c_{\alpha}\left(1-|z|^{2}\right)^{\alpha} d \nu(z), z \in B$. Here $c_{\alpha}$ is a positive constant such that $v_{\alpha}(B)=1$. Let $H(B)$ denote the space of all holomorphic functions in $B$. For a twice differentiable, nondecreasing, nonnegative strongly convex function $\varphi$ on the real line $\mathbb{R}$, define the Bergman-Orlicz space $A_{\varphi}\left(\nu_{\alpha}\right)$ by

$$
A_{\varphi}\left(\nu_{\alpha}\right)=\left\{f \in H(B): \int_{B} \varphi(\log |f|) d v_{\alpha}<\infty\right\} .
$$
\end{abstract}

In this paper we prove that a function $f \in H(B)$ is in $A_{\varphi}\left(\nu_{\alpha}\right)$ if and only if

$$
\int_{B} \varphi^{\prime \prime}(\log |f(z)|) \frac{|\mathscr{R} f(z)|^{2}}{|z|^{2}|f(z)|^{2}}\left(1-|z|^{2}\right)^{2} d v_{\alpha}(z)<\infty,
$$

where $\mathscr{R} f(z)=\sum_{j=1}^{n} z_{j} \partial f(z) / \partial z_{j}$ is the radial derivative of $f$.

2000 Mathematics subject classification: primary 32A36; secondary 32A35, $32 \mathrm{~A} 37$.

\section{Introduction}

Let $n \geq 1$ be a fixed integer. Let $H(B)$ denote the space of all holomorphic functions in the unit ball $B \equiv B_{n}$ of the complex $n$-dimensional Euclidean space $\mathbb{C}^{n}$. Let $v$ denote the normalized Lebesgue measure on $B$. For each $\alpha \in(-1, \infty)$, we set $c_{\alpha}=\Gamma(n+\alpha+1) /\{\Gamma(n+1) \Gamma(\alpha+1)\}$ and $d \nu_{\alpha}(z)=c_{\alpha}\left(1-|z|^{2}\right)^{\alpha} d \nu(z), z \in B$. Note that $v_{\alpha}(B)=1$. Let $\mathscr{S} T^{2}(\mathbb{R})$ denote the class of those nondecreasing convex functions $\varphi:[-\infty, \infty) \rightarrow[0, \infty)$ which are twice differentiable in $(-\infty, \infty)$ and satisfy the

(C) 2003 Australian Mathematical Society $1446-7887 / 03 \$$ A2.00+0.00 
growth condition $\lim _{t \rightarrow \infty} \varphi(t) / t=\infty$. For each $\alpha \in(-1, \infty)$ and $\varphi \in \mathscr{S} T^{2}(\mathbb{R})$, we define the weighted Bergman-Orlicz space $A_{\varphi}\left(\nu_{\alpha}\right)$ by

$$
A_{\varphi}\left(v_{\alpha}\right)=\left\{f \in H(B):\|f\|_{A_{\varphi}\left(\nu_{\alpha}\right)} \equiv \int_{B} \varphi(\log |f|) d \nu_{\alpha}<\infty\right\} .
$$

The Hardy-Orlicz space $H_{\varphi}(B)$ is as usual defined by

$$
H_{\varphi}(B)=\left\{f \in H(B):\|f\|_{H_{\varphi}(B)} \equiv \sup _{0 \leq r<1} \int_{S} \varphi\left(\log \left|f_{r}\right|\right) d \sigma<\infty\right\},
$$

where $\sigma$ is the normalized Lebesgue measure on the unit sphere $S \equiv \partial B$ and $f_{r}(z)=$ $f(r z)$ for $0 \leq r<1, z \in \mathbb{C}^{n}$ with $r z \in B$. In 1985, Beatrous and Burbea [1] gave the following characterization of the Bergman spaces $A^{p}\left(v_{\alpha}\right) \equiv H(B) \cap L^{p}\left(v_{\alpha}\right)$, $0<p<\infty$.

THEOREM 1.1 (Beatrous and Burbea). Let $f \in H(B) \backslash\{0\}, \alpha \in(-1, \infty)$ and let $0<p<\infty$. Then $f \in A^{p}\left(\nu_{\alpha}\right)$ if and only if

$$
\int_{B}|f(z)|^{p} \frac{|\mathscr{R} f(z)|^{2}}{|z|^{2}|f(z)|^{2}}\left(1-|z|^{2}\right)^{2} d v_{\alpha}(z)<\infty,
$$

where $\mathscr{R} f(z)=\sum_{j=1}^{n} z_{j} \partial f(z) / \partial z_{j}$ is the radial derivative of $f$.

This characterization of the weighted Bergman spaces is of the same type as that of the Hardy spaces by Yamashita [8] and Stoll [6]. The purpose of the present paper is to give the characterization of the weighted Bergman-Orlicz spaces $A_{\varphi}\left(v_{\alpha}\right)$, $\varphi \in \mathscr{S} T^{2}(\mathbb{R}),-1<\alpha<\infty$, which is of the Beatrous-Burbea's type. Our main result (Section 4, Theorem 4.1) contains, as the limiting case $\alpha=-1$, a characterization of the Hardy-Orlicz spaces $H_{\varphi}(B), \varphi \in \mathscr{S} T^{2}(\mathbb{R})$.

THEOREM 1.2. Let $\varphi \in \mathscr{S} T^{2}(\mathbb{R})$ and $f \in H(B) \backslash\{0\}$. Then $f \in H_{\varphi}(B)$ if and only if

$$
\int_{B} \varphi^{\prime \prime}(\log |f(z)|) \frac{|\mathscr{R} f(z)|^{2}}{|z|^{2}|f(z)|^{2}}\left(1-|z|^{2}\right) d \nu(z)<\infty .
$$

This characterization is a little bit different from that of by Ouyang and Riihentaus [2].

THEOREM 1.3 (Ouyang and Riihentaus). Let $\varphi \in \mathscr{S} T^{2}(\mathbb{R})$ and $f \in H(B) \backslash\{0\}$. Then $f \in H_{\varphi}(B)$ if and only if

$$
\int_{B} \varphi^{\prime \prime}(\log |f(z)|) \frac{|\nabla f(z)|^{2}}{|f(z)|^{2}}\left(1-|z|^{2}\right) d \nu(z)<\infty,
$$

where $|\nabla f(z)|^{2}=\sum_{j=1}^{n}\left|\partial f(z) / \partial z_{j}\right|^{2}$. 
We note that the results of Stoll [5] and Ouyang-Riihentaus [2] hold for more general domains in $\mathbb{C}$ and $\mathbb{C}^{n}$ than for $\mathbb{D}$ and $B$, respectively.

\section{Notation}

Let $\mathscr{M}$ denote the group of biholomorphic maps of $B$ onto itself. For each $a \in B$, let $\varphi_{a} \in \mathscr{M}$ be the involution described in [3, page 25]. Let $\lambda$ be the measure on $B$ defined by

$$
d \lambda(z)=\frac{1}{\left(1-|z|^{2}\right)^{n+1}} d \nu(z), \quad z \in B
$$

Then $\lambda$ is the invariant volume measure induced by the Bergman metric on $B$. Thus

$$
\int_{B} f d \lambda=\int_{B}(f \circ \psi) d \lambda
$$

for each $f \in L^{1}(\lambda)$ and all $\psi \in \mathscr{M}$ ([3, Theorem 2.2.6]). For $f \in C^{2}(B)$ and $a \in B$, define

$$
\tilde{\Delta} f(a)=\frac{1}{n+1} \Delta\left(f \circ \varphi_{a}\right)(0),
$$

where $\Delta \equiv 4 \sum_{j=1}^{n} \partial^{2} / \partial z_{j} \partial \bar{z}_{j}$ is the ordinary Laplacian. Then as in [3, Theorem 4.1.3],

$$
\tilde{\Delta} f(a)=\frac{4}{n+1}\left(1-|a|^{2}\right) \sum_{i, j=1}^{n}\left(\delta_{i j}-a_{i} \bar{a}_{j}\right) \frac{\partial^{2} f}{\partial z_{i} \partial \bar{z}_{j}}(a) .
$$

The operator $\tilde{\Delta}$ is invariant under $\mathscr{M}$, that is, $\tilde{\Delta}(f \circ \psi)=(\tilde{\Delta} f) \circ \psi$ for all $\psi \in \mathscr{M}$ ([3, Theorem 4.1.2]). Let $\tilde{\nabla}$ denote the gradient with respect to the Bergman metric on $B$ ([7, page 27]). Then as in [7, page 30], for $f \in H(B)$

$$
|\tilde{\nabla} f(a)|^{2}=\frac{2}{n+1}\left(1-|a|^{2}\right)\left[\sum_{j=1}^{n}\left|\frac{\partial f}{\partial z_{j}}(a)\right|^{2}-\left|\sum_{j=1}^{n} a_{j} \frac{\partial f}{\partial z_{j}}(a)\right|^{2}\right], \quad a \in B .
$$

An upper semicontinuous function $u: B \rightarrow[-\infty, \infty), u \neq \equiv-\infty$, is said to be $\mathscr{M}$-subharmonic if for each $a \in B$

$$
u(a) \leq \int_{S} u\left(\varphi_{a}(r \zeta)\right) d \sigma(\zeta), \quad 0<r<1 .
$$

A continuous function $u$ defined in $B$ is said to be $\mathscr{M}$-harmonic if equality holds in the above inequality. A function $u$ in $B$ is said to be $\mathscr{M}$-superharmonic if $-u$ is $\mathscr{M}$-subharmonic. 
As in [7, Section 6.2], the invariant Green's function on $B$ is given by $G(z, a)=$ $g\left(\varphi_{a}(z)\right)$ for $(z, a) \in B \times B$, where

$$
g(z)=\frac{n+1}{2 n} \int_{|z|}^{1}\left(1-t^{2}\right)^{n-1} t^{-2 n+1} d t .
$$

Note that $g$ is $\mathscr{M}$-harmonic in $B \backslash\{0\}$, and $\mathscr{M}$-superharmonic in $B$. Let $f$ be an $\mathscr{M}$-subharmonic function in $B$. The Riesz measure of $f$ is the non-negative regular Borel measure $\mu_{f}$ in $B$ which satisfies

$$
\int_{B} \psi d \mu_{f}=\int_{B} f \tilde{\Delta} \psi d \lambda
$$

for all $\psi \in C_{c}^{2}(B)$. Here $C_{c}^{2}(B)$ is the class of twice continuously differentiable functions in $B$ with compact support. If $f$ is in $C^{2}(B)$, then by Green's identity [7, Proposition 3.1] $d \mu_{f}=\tilde{\Delta} f d \lambda$.

In the case $n=1, B_{1} \equiv \mathbb{D}$ is the unit disc and $S_{1} \equiv \mathbb{T}$ is the unit circle in the complex plane $\mathbb{C}$. Moreover, $g(z)=\log (1 /|z|)$ and $(\tilde{\Delta} f)(z)=\frac{1}{2}\left(1-|z|^{2}\right)^{2}(\Delta f)(z)$ for $f \in C^{2}(\mathbb{D})$ and $z \in \mathbb{D}$.

\section{Preliminaries}

According to [1, page 41], we introduce positive functions $\left\{K_{\alpha}:-1 \leq \alpha<\infty\right\}$ defined in the interval $(0,1)$ as follows. For $t \in(0,1)$,

$$
K_{\alpha}(t)=2 n c_{\alpha} \int_{t}^{1} \rho^{2 n-1}\left(1-\rho^{2}\right)^{\alpha} \log \frac{\rho}{t} d \rho \quad \text { if } \alpha>-1
$$

and $K_{-1}(t)=\log (1 / t)$. The following lemma is easily verified. (See, for example, [1, Proposition 2.3].)

LEMMA 3.1. The following two inequalities hold.

$$
0<1-t^{2}<2 K_{-1}(t) \quad(0<t<1), \quad K_{-1}(t)<1-t^{2} \quad(1 / 2<t<1) .
$$

For each $\alpha \in(-1, \infty)$, there exist two positive numbers $c_{\alpha 1}$ and $c_{\alpha 2}$ such that

$$
c_{\alpha 1}\left(1-t^{2}\right)^{\alpha+2} \leq K_{\alpha}(t) \leq c_{\alpha 2}\left(1-t^{2}\right)^{\alpha+2} \quad(0<t<1) .
$$

For $f \in H(B)$ we denote the zero set of $f$ by $Z(f) \equiv\{z \in B: f(z)=0\}$. A simple computation shows the following lemma.

Lemma 3.2. Suppose $\varphi \in \mathscr{S} T^{2}(\mathbb{R})$ and $f \in H(\mathbb{D}) \backslash\{0\}$. Put $v=\varphi(\log |f|)$ in $\mathbb{D}$. Then 
(1) $\Delta v=\varphi^{\prime \prime}(\log |f|)\left|f^{\prime}\right|^{2} /|f|^{2}$ in $\mathbb{D} \backslash Z(f)$.

(2) $(\tilde{\Delta} v)(z)=\frac{1}{2}\left(1-|z|^{2}\right)^{2}(\Delta v)(z)=\frac{1}{2}\left(1-|z|^{2}\right)^{2} \varphi^{\prime \prime}(\log |f(z)|)\left|f^{\prime}(z)\right|^{2} /|f(z)|^{2}$ for $z \in \mathbb{D} \backslash Z(f)$.

LEMma 3.3. Suppose $\varphi \in \mathscr{S} T^{2}(\mathbb{R})$ and $f \in H(\mathbb{D}) \backslash\{0\}$. Put $v=\varphi(\log |f|)$ in $\mathbb{D}$. Then the Riesz measure $\mu_{v}$ is given by

$$
\begin{aligned}
d \mu_{v}(z) & =\frac{1}{2} \varphi^{\prime \prime}(\log |f(z)|) \frac{\left|f^{\prime}(z)\right|^{2}}{|f(z)|^{2}} d \nu(z) \\
& =\frac{1}{2} \Delta(v \mid \mathbb{D} \backslash Z(f))(z) d v(z)=\frac{1}{2} \tilde{\Delta}(v \mid \mathbb{D} \backslash Z(f))(z) d \lambda(z)
\end{aligned}
$$

for $z \in \mathbb{D}$. Here we use the convention that the right hand sides of these equations are defined to be 0 in $Z(f)$.

PROOF. The first equation follows from [5, (3.1), pages 1035-1037] and the two remaining equations follow then from Lemma 3.2.

LEMMA 3.4. Suppose $\varphi \in \mathscr{S} T^{2}(\mathbb{R})$ and $f \in H_{\varphi}(\mathbb{D}) \backslash\{0\}$. Put $v=\varphi(\log |f|)$ in $\mathbb{D}$. Then $v$ has a harmonic majorant in $\mathbb{D}$. And the least harmonic majorant of $v$ is the Poisson integral $P\left[v^{*}\right]$ of $v^{*}=\varphi\left(\log \left|f^{*}\right|\right)$. Here $f^{*}(\zeta)=\lim _{r \uparrow 1} f(r \zeta)$ for almost every $\zeta \in \mathbb{T}$ and

$$
P\left[v^{*}\right](z)=\int_{\mathbf{T}} \frac{1-|z|^{2}}{|1-z \bar{\zeta}|^{2}} v^{*}(\zeta) d \sigma(\zeta) \quad(z \in \mathbb{D})
$$

Proof. First, one sees easily that $f \in N_{*}(\mathbb{D}) \subset N(\mathbb{D})$ (for the definition of the Smirnov class $N_{*}(\mathbb{D})$ see, for example, [4, page 85] or [3, 19.1.11, page 407]). Then by $[3,5.6 .4$. Theorem, page 85$] f^{*}$ is defined (almost everywhere on $\mathbb{T}$ ) and the least harmonic majorant of $\log |f|$ is $u=P\left[\log \left|f^{*}\right| d \sigma+d \gamma\right]$, where $\gamma$ is a singular measure on $\mathbb{T}$. By [4, Theorem 2, page 84] the boundary measure of $v=\varphi(\log |f|)$ is $\varphi\left(\log \left|f^{*}\right|\right) d \sigma$, hence the least harmonic majorant of $v$ is $P\left[\varphi\left(\log \left|f^{*}\right|\right)\right]$.

LEMMA 3.5. Suppose $\varphi \in \mathscr{S} T^{2}(\mathbb{R})$ and $f \in H_{\varphi}(\mathbb{D}) \backslash\{0\}$. Then

$$
\|f\|_{H_{\varphi}(\mathbb{D})}=\varphi(\log |f(0)|)+\frac{1}{2} \int_{\mathbb{D}} \varphi^{\prime \prime}(\log |f(z)|) \frac{\left|f^{\prime}(z)\right|^{2}}{|f(z)|^{2}} \log \frac{1}{|z|} d \nu(z) .
$$

PROof. Since $\lim _{t \rightarrow \infty} \varphi(t) / t=\infty$, it holds that

$$
\|f\|_{H_{\varphi}(\mathbb{D})}=\int_{\mathbb{T}} \varphi\left(\log \left|f^{*}\right|\right) d \sigma=P\left[\varphi\left(\log \left|f^{*}\right|\right)\right](0) .
$$


With Lemma 3.3 and Lemma 3.4, the Riesz decomposition theorem ([7, Corollary 6.11]) implies that

$$
\begin{aligned}
\varphi(\log |f(z)|) & =P\left[\varphi\left(\log \left|f^{*}\right|\right)\right](z)-\int_{\mathbb{D}} G(z, w) \tilde{\Delta}(\{\varphi(\log |f|)\})(w) d \lambda(w) \\
& =P\left[\varphi\left(\log \left|f^{*}\right|\right)\right](z)-\frac{1}{2} \int_{\mathbb{D}} G(z, w) \varphi^{\prime \prime}(\log |f(w)|) \frac{\left|f^{\prime}(w)\right|^{2}}{|f(w)|^{2}} d \nu(w)
\end{aligned}
$$

for all $z \in \mathbb{D}$. Putting $z=0$ in the above equations, we obtain the lemma.

For $\varphi \in \mathscr{S} T^{2}(\mathbb{R})$ and $f \in H_{\varphi}(B) \backslash\{0\}$, we define

$$
f_{\varphi}^{\sharp}(z)=\varphi^{\prime \prime}(\log |f(z)|) \frac{|(\mathscr{R} f)(z)|^{2}}{|f(z)|^{2}}|z|^{-2} \quad(z \in B \backslash[Z(f) \cup\{0\}]) .
$$

Let $A(B)$ denote the ball algebra: $A(B) \equiv C(\bar{B}) \cap H(B)$.

Lemma 3.6. Suppose $\varphi \in \mathscr{S} T^{2}(\mathbb{R})$ and $f \in A(B) \backslash\{0\}$. Then

$$
\|f\|_{H_{\varphi}(B)}=\varphi(\log |f(0)|)+\frac{1}{2 n} \int_{B} f_{\varphi}^{\sharp}(z)|z|^{-2(n-1)} \log \frac{1}{|z|} d v(z) .
$$

Proof. Almost every $\zeta \in S, f_{\zeta} \in A(\mathbb{D}) \backslash\{0\} \subset H_{\varphi}(\mathbb{D}) \backslash\{0\}$. Here $A(\mathbb{D}) \equiv$ $C(\overline{\mathbb{D}}) \cap H(\mathbb{D})$ and $f_{\zeta}(t)=f(t \zeta)$ for $t \in \overline{\mathbb{D}}$. By Lemma 3.5,

$$
\begin{aligned}
\left\|f_{\zeta}\right\|_{H_{\varphi}(\mathbb{D})}-\varphi(\log |f(0)|) & =\frac{1}{2} \int_{\mathbb{D}} \varphi^{\prime \prime}\left(\log \left|f_{\zeta}(t)\right|\right) \frac{\left|\left(f_{\zeta}\right)^{\prime}(t)\right|^{2}}{\left|f_{\zeta}(t)\right|^{2}} \log \frac{1}{|t|} d \nu_{1}(t) \\
& =\frac{1}{2} \int_{\mathbb{D}} \varphi^{\prime \prime}(\log |f(t \zeta)|)|t|^{-2} \frac{|(\mathscr{R} f)(t \zeta)|^{2}}{|f(t \zeta)|^{2}} \log \frac{1}{|t|} d \nu_{1}(t) \\
& =\frac{1}{2} \int_{\mathbb{D}} f_{\varphi}^{\ddagger}(t \zeta) \log \frac{1}{|t|} d \nu_{1}(t) .
\end{aligned}
$$

On the other hand, the assumption $f \in A(B)$ implies

$$
\begin{aligned}
\|f\|_{H_{\varphi}(B)} & =\int_{S} \varphi(\log |f|) d \sigma=\int_{S} d \sigma(\zeta) \frac{1}{2 \pi} \int_{0}^{2 \pi} \varphi\left(\log \left|f\left(e^{i \theta} \zeta\right)\right|\right) d \theta \\
& =\int_{S} d \sigma(\zeta) \frac{1}{2 \pi} \int_{0}^{2 \pi} \varphi\left(\log \left|f_{\zeta}\left(e^{i \theta}\right)\right|\right) d \theta=\int_{S}\left\|f_{\zeta}\right\|_{H_{\varphi}(\mathbb{D})} d \sigma(\zeta)
\end{aligned}
$$


We used here the formula in $[3,1.4 .7$. Proposition (1), page 15]. Hence we have

$$
\begin{aligned}
& \|f\|_{H_{\varphi}(B)}-\varphi(\log |f(0)|)=\int_{S}\left\{\left\|f_{\zeta}\right\|_{H_{\varphi}(\mathbb{D})}-\varphi(\log |f(0)|)\right\} d \sigma(\zeta) \\
& =\int_{S} d \sigma(\zeta) \frac{1}{2} \int_{\mathbb{D}} f_{\varphi}^{\sharp}(t \zeta) \log \frac{1}{|t|} d \nu_{1}(t) \\
& =\frac{1}{2} \int_{S} d \sigma(\zeta) 2 \int_{0}^{1} r d r \frac{1}{2 \pi} \int_{0}^{2 \pi} f_{\varphi}^{\sharp}\left(r e^{i \theta} \zeta\right) \log \frac{1}{r} d \theta \\
& =\int_{0}^{1} r \log \frac{1}{r} d r \int_{S} d \sigma(\zeta) \frac{1}{2 \pi} \int_{0}^{2 \pi} f_{\varphi}^{\sharp}\left(r e^{i \theta} \zeta\right) d \theta \\
& =\frac{1}{2 n} 2 n \int_{0}^{1} r \log \frac{1}{r} d r \int_{S} f_{\varphi}^{\sharp}(r \zeta) d \sigma(\zeta) \\
& =\frac{1}{2 n} 2 n \int_{0}^{1} r^{2 n-1} d r \int_{S}|r \zeta|^{-2(n-1)} \log \frac{1}{|r \zeta|} f_{\varphi}^{\sharp}(r \zeta) d \sigma(\zeta) \\
& =\frac{1}{2 n} \int_{B}|z|^{-2(n-1)} \log \frac{1}{|z|} f_{\varphi}^{\sharp}(z) d v(z) \text {. }
\end{aligned}
$$

LEMMA 3.7. Suppose $\varphi \in \mathscr{S} T^{2}(\mathbb{R}), f \in H(B) \backslash\{0\}$ and $0<r<1$. Then

$$
\left\|f_{r}\right\|_{H_{\varphi}(B)}=\varphi(\log |f(0)|)+\frac{1}{2 n} \int_{r B} f_{\varphi}^{\sharp}(z)|z|^{-2(n-1)} \log \frac{r}{|z|} d \nu(z),
$$

where $r B=\left\{z \in \mathbb{C}^{n}:|z|<r\right\}$.

PROOF. Since $f_{r} \in A(B) \backslash\{0\}$, Lemma 3.6 implies that

$$
\left\|f_{r}\right\|_{H_{\varphi}(B)}-\varphi(\log |f(0)|)=\frac{1}{2 n} \int_{B}\left(f_{r}\right)_{\varphi}^{\sharp}(z)|z|^{-2(n-1)} \log \frac{1}{|z|} d \nu(z) .
$$

By the change of variables $w=r z, z \in B$, we have

$$
\begin{aligned}
\left\|f_{r}\right\|_{H_{\varphi}(B)}-\varphi(\log |f(0)|) & =\frac{1}{2 n} \int_{r B} f_{\varphi}^{\sharp}(w) \frac{r^{2 n}}{|w|^{2(n-1)}} \log \frac{r}{|w|} r^{-2 n} d \nu(w) \\
& =\frac{1}{2 n} \int_{r B} f_{\varphi}^{\sharp}(w)|w|^{-2(n-1)} \log \frac{r}{|w|} d \nu(w) .
\end{aligned}
$$

LEMMA 3.8. Suppose $-1<\alpha<\infty, \varphi \in \mathscr{S} T^{2}(\mathbb{R})$ and $f \in H(B) \backslash\{0\}$. Then

$$
\|f\|_{A_{\varphi}\left(\nu_{\alpha}\right)}=\varphi(\log |f(0)|)+\frac{1}{2 n} \int_{B} f_{\varphi}^{\sharp}(z)|z|^{-2(n-1)} K_{\alpha}(|z|) d \nu(z) .
$$


Proof. Using Lemma 3.7, Fubini's theorem and the definition of the function $K_{\alpha}$, we have

$$
\begin{aligned}
\|f\|_{A_{\varphi}\left(\nu_{\alpha}\right)}-\varphi(\log |f(0)|) \\
\quad=\int_{B} \varphi(\log |f|) d \nu_{\alpha}-\varphi(\log |f(0)|) \\
\quad=c_{\alpha} 2 n \int_{0}^{1} r^{2 n-1}\left(1-r^{2}\right)^{\alpha} d r \int_{S} \varphi\left(\log \left|f_{r}(\zeta)\right|\right) d \sigma(\zeta)-\varphi(\log |f(0)|) \\
\quad=2 n c_{\alpha} \int_{0}^{1} r^{2 n-1}\left(1-r^{2}\right)^{\alpha}\left\{\left\|f_{r}\right\|_{H_{\varphi}(B)}-\varphi(\log |f(0)|)\right\} d r \\
=2 n c_{\alpha} \int_{0}^{1} r^{2 n-1}\left(1-r^{2}\right)^{\alpha} d r \frac{1}{2 n} \int_{r B} f_{\varphi}^{\sharp}(z)|z|^{-2(n-1)} \log \frac{r}{|z|} d \nu(z) \\
=c_{\alpha} \int_{B} f_{\varphi}^{\sharp}(z)|z|^{-2(n-1)} d \nu(z) \int_{|z|}^{1} r^{2 n-1}\left(1-r^{2}\right)^{\alpha} \log \frac{r}{|z|} d r \\
=\frac{1}{2 n} \int_{B} f_{\varphi}^{\sharp}(z)|z|^{-2(n-1)} K_{\alpha}(|z|) d \nu(z) .
\end{aligned}
$$

LEMma 3.9. Suppose $\varphi \in \mathscr{S} T^{2}(\mathbb{R})$ and $f \in H(B) \backslash\{0\}$. Then

$$
\|f\|_{H_{\varphi}(B)}=\varphi(\log |f(0)|)+\frac{1}{2 n} \int_{B} f_{\varphi}^{\sharp}(z)|z|^{-2(n-1)} K_{-1}(|z|) d \nu(z) .
$$

PROOF. For any $r \in(0,1)$, by Lemma 3.7

$$
\left\|f_{r}\right\|_{H_{\varphi}(B)}=\varphi(\log |f(0)|)+\frac{1}{2 n} \int_{r B} f_{\varphi}^{\sharp}(z)|z|^{-2(n-1)} \log \frac{r}{|z|} d \nu(z) .
$$

Using the subharmonicity of the function $\varphi(\log |f|)$ and the monotone convergence theorem, we have

$$
\begin{aligned}
\|f\|_{H_{\varphi}(B)}-\varphi(\log |f(0)|) & =\lim _{r \uparrow 1}\left\|f_{r}\right\|_{H_{\varphi}(B)}-\varphi(\log |f(0)|) \\
& =\frac{1}{2 n} \int_{B} f_{\varphi}^{\sharp}(z)|z|^{-2(n-1)} \log \frac{1}{|z|} d \nu(z) \\
& =\frac{1}{2 n} \int_{B} f_{\varphi}^{\sharp}(z)|z|^{-2(n-1)} K_{-1}(|z|) d \nu(z) .
\end{aligned}
$$

For the sake of convenience we define $A_{\varphi}\left(\nu_{-1}\right) \equiv H_{\varphi}(B)$ for $\varphi \in \mathscr{S} T^{2}(\mathbb{R})$, and $\|f\|_{A_{\varphi}\left(\nu_{-1}\right)} \equiv\|f\|_{H_{\varphi}(B)}$ for $f \in H(B) \backslash\{0\}$. Then we can unify Lemma 3.8 and Lemma 3.9 in the following form.

LEMMA 3.10. Suppose $-1 \leq \alpha<\infty, \varphi \in \mathscr{S} T^{2}(\mathbb{R})$ and $f \in H(B) \backslash\{0\}$. Then

$$
\|f\|_{A_{\varphi}\left(v_{\mathbf{\alpha}}\right)}=\varphi(\log |f(0)|)+\frac{1}{2 n} \int_{B} f_{\varphi}^{\sharp}(z)|z|^{-2(n-1)} K_{\alpha}(|z|) d \nu(z) .
$$


This is a generalization of [1, Theorem 3.3].

For $\zeta \in S$ and $\beta \in(1, \infty)$ we define the Korányi approach region $D_{\beta}(\zeta)$ by

$$
D_{\beta}(\zeta) \equiv\left\{z \in \mathbb{C}^{n}:|1-\langle z, \zeta\rangle|<\beta\left(1-|z|^{2}\right) / 2\right\}
$$

Let $-1 \leq \alpha<\infty, \varphi \in \mathscr{S} T^{2}(\mathbb{R})$ and $1<\beta<\infty$. For $f \in H(B) \backslash\{0\}$ and $\zeta \in S$, we define

and

$$
L_{\varphi}(\zeta: f, \alpha, \beta) \equiv \int_{D_{\beta}(\zeta)} f_{\varphi}^{\sharp}(z)\left(1-|z|^{2}\right)^{\alpha+2-n} d \nu(z)
$$

$$
\mathscr{L}_{\varphi}(f, \alpha, \beta) \equiv \int_{S} L_{\varphi}(\zeta: f, \alpha, \beta) d \sigma(\zeta)
$$

For any $\beta \in(1, \infty)$ and $z \in B$, we define

$$
Q_{\beta}(z) \equiv\left\{\zeta \in S:|1-\langle z, \zeta)|<\beta\left(1-|z|^{2}\right) / 2\right\}
$$

and $\omega_{\beta}(z) \equiv \sigma\left(Q_{\beta}(z)\right)$. We note that $\omega_{\beta}$ is a radial function in $B$ :

$$
\omega_{\beta}(U z)=\omega_{\beta}(z) \quad(z \in B, U \in \mathscr{U})
$$

where $\mathscr{U}$ is the unitary group of $\mathbb{C}^{n}$. Hence there exists a function $F_{\beta}$ defined in the interval $[0,1)$ such that $F_{\beta}(|z|)=\omega_{\beta}(z)(z \in B)$. For any $\beta \in(1, \infty)$, we define $r_{\beta} \equiv \max \{0,(2-\beta) / \beta\}$ and $G_{\beta}(r) \equiv F_{\beta}(r) r^{2(n-1)}\left(1-r^{2}\right)^{-n}(0 \leq r<1)$.

LeMma 3.11. Let $\beta \in(1, \infty)$. Then $G_{\beta}(r)=0$ if $0 \leq r \leq r_{\beta}$ and $G_{\beta}(r)>0$ if $r_{\beta}<r<1$. Moreover, $G_{\beta}$ is a continuous bounded function in the interval $[0,1)$.

Proof. See [1, Proposition 4.2].

LEMMA 3.12. Let $-1 \leq \alpha<\infty, 1<\beta<\infty$ and $r_{\beta}<r<1$. Suppose $\varphi \in \mathscr{S} T^{2}(\mathbb{R})$ and $f \in H(B) \backslash\{0\}$. Then

$$
\begin{aligned}
& \left\{\inf _{r<t<1} G_{\beta}(t)\right\} \int_{B \backslash \bar{B}} f_{\varphi}^{\sharp}(z)|z|^{-2(n-1)}\left(1-|z|^{2}\right)^{\alpha+2} d \nu(z) \\
& \quad \leq \mathscr{L}_{\varphi}(f, \alpha, \beta) \leq\left\{\sup _{r_{\beta}<t<1} G_{\beta}(t)\right\} \int_{B \backslash r_{\beta} \bar{B}} f_{\varphi}^{\sharp}(z)|z|^{-2(n-1)}\left(1-|z|^{2}\right)^{\alpha+2} d \nu(z) .
\end{aligned}
$$


Proof. (See [1, Theorem 4.3].) By the definition of $\mathscr{L}_{\varphi}$, Fubini's theorem and Lemma 3.11

$$
\begin{aligned}
\mathscr{L}_{\varphi}(f, \alpha, \beta) & =\int_{S} L_{\varphi}(\zeta: f, \alpha, \beta) d \sigma(\zeta) \\
& =\int_{S} d \sigma(\zeta) \int_{D_{\beta}(\zeta)} f_{\varphi}^{\sharp}(z)\left(1-|z|^{2}\right)^{\alpha+2-n} d \nu(z) \\
& =\int_{S} d \sigma(\zeta) \int_{B} \chi_{D_{\beta}(\zeta)}(z) f_{\varphi}^{\sharp}(z)\left(1-|z|^{2}\right)^{\alpha+2-n} d \nu(z) \\
& =\int_{B} f_{\varphi}^{\sharp}(z)\left(1-|z|^{2}\right)^{\alpha+2-n} d \nu(z) \int_{S} \chi_{Q_{\beta}(z)}(\zeta) d \sigma(\zeta) \\
& =\int_{B} f_{\varphi}^{\sharp}(z)\left(1-|z|^{2}\right)^{\alpha+2-n} \sigma\left(Q_{\beta}(z)\right) d \nu(z) \\
& =\int_{B} f_{\varphi}^{\sharp}(z)\left(1-|z|^{2}\right)^{\alpha+2-n} F_{\beta}(|z|) d \nu(z) \\
& =\int_{B} f_{\varphi}^{\sharp}(z)\left(1-|z|^{2}\right)^{\alpha+2-n} G_{\beta}(|z|)|z|^{-2(n-1)}\left(1-|z|^{2}\right)^{n} d \nu(z) \\
& =\int_{B} f_{\varphi}^{\sharp}(z)\left(1-|z|^{2}\right)^{\alpha+2}|z|^{-2(n-1)} G_{\beta}(|z|) d \nu(z) \\
& =\int_{B \backslash r_{\beta} \bar{B}} f_{\varphi}^{\sharp}(z)\left(1-|z|^{2}\right)^{\alpha+2}|z|^{-2(n-1)} G_{\beta}(|z|) d \nu(z)
\end{aligned}
$$

Since $B \backslash r \bar{B} \subset B \backslash r_{\beta} \bar{B}$, the above equations prove the lemma.

Lemma 3.13. Let $-1 \leq \alpha<\infty, 0<r<1$ and $z \in r B$. Then it holds that

$$
K_{\alpha}(|z|) \leq K_{\alpha}(|z| / r)+\log (1 / r)
$$

PROOF. See [1, pages 48-49].

LEMMA 3.14. Let $-1 \leq \alpha<\infty$ and $0<r<1$. Suppose $\varphi \in \mathscr{S} T^{2}(\mathbb{R})$ and $f \in H(B) \backslash\{0\}$. Then

$$
\int_{r B} f_{\varphi}^{\sharp}(z)|z|^{-2(n-1)} d \nu(z)<\infty
$$

and

$$
\int_{r B} f_{\varphi}^{\sharp}(z)|z|^{-2(n-1)} K_{\alpha}(|z|) d \nu(z)<\infty .
$$

Proof. (See [1, Lemma 5.1].) Choose a number $r^{\prime}$ so that $r<r^{\prime}<1$. Since 
$f_{r} \in A(B) \backslash\{0\}$, by Lemma 3.7, we have

$$
\begin{aligned}
\int_{r B} f_{\varphi}^{\sharp}(z)|z|^{-2(n-1)} K_{-1}\left(\frac{|z|}{r}\right) d \nu(z) & =\int_{r B} f_{\varphi}^{\sharp}(z)|z|^{-2(n-1)} \log \frac{r}{|z|} d \nu(z) \\
& =2 n\left\{\left\|f_{r}\right\|_{H_{\varphi}(B)}-\varphi(\log |f(0)|)\right\}<\infty
\end{aligned}
$$

and also $\int_{r^{\prime} B} f_{\varphi}^{\sharp}(z)|z|^{-2(n-1)} \log \left(r^{\prime} /|z|\right) d \nu(z)<\infty$. Hence

$$
\begin{aligned}
\int_{r B} f_{\varphi}^{\sharp}(z)|z|^{-2(n-1)} d \nu(z) & \leq \int_{r B} f_{\varphi}^{\sharp}(z)|z|^{-2(n-1)} \frac{\log \left(r^{\prime} /|z|\right)}{\log \left(r^{\prime} / r\right)} d \nu(z) \\
& \leq \frac{1}{\log \left(r^{\prime} / r\right)} \int_{r^{\prime} B} f_{\varphi}^{\sharp}(z)|z|^{-2(n-1)} \log \frac{r^{\prime}}{|z|} d \nu(z)<\infty .
\end{aligned}
$$

This proves (1). In the case $-1<\alpha$, by Lemma 3.8

$$
\begin{aligned}
\int_{r B} f_{\varphi}^{z}(z)|z|^{-2(n-1)} K_{\alpha}\left(\frac{|z|}{r}\right) d \nu(z) & =\int_{B} r^{2} f_{\varphi}^{\sharp}(r z)|z|^{-2(n-1)} K_{\alpha}(|z|) d \nu(z) \\
& =\int_{B}\left(f_{r}\right)_{\varphi}^{\sharp}(z)|z|^{-2(n-1)} K_{\alpha}(|z|) d \nu(z) \\
& =2 n\left\{\left\|f_{r}\right\|_{A_{\varphi}\left(v_{\alpha}\right)}-\varphi(\log |f(0)|)\right\}<\infty .
\end{aligned}
$$

By this, (3), (1) and Lemma 3.13 we obtain (2).

\section{Main result}

THEOREM 4.1. Let $-1 \leq \alpha<\infty, \varphi \in \mathscr{S} T^{2}(\mathbb{R})$ and $f \in H(B) \backslash\{0\}$. Then the following statements are equivalent:

(a) $f \in A_{\varphi}\left(v_{\alpha}\right)$ if $-1<\alpha<\infty$. $f \in H_{\varphi}$ (B) if $\alpha=-1$.

(b) $\int_{B} f_{\varphi}^{\sharp}(z)\left(1-|z|^{2}\right)^{\alpha+2} d v(z)<\infty$.

(c) $\mathscr{L}_{\varphi}(f, \alpha, \beta)<\infty$ for any $\beta \in(1, \infty)$.

(d) $\mathscr{L}_{\varphi}(f, \alpha, \beta)<\infty$ for some $\beta \in(1, \infty)$.

PROOF. (a) implies (b). Using Lemma 3.1 and Lemma 3.10, we have

$$
\begin{aligned}
\int_{B} f_{\varphi}^{z}(z)\left(1-|z|^{2}\right)^{\alpha+2} d \nu(z) & \leq \frac{1}{c_{\alpha 1}} \int_{B} f_{\varphi}^{\sharp}(z) K_{\alpha}(|z|) d v(z) \\
& \leq \frac{1}{c_{\alpha 1}} \int_{B} f_{\varphi}^{\sharp}(z)|z|^{-2(n-1)} K_{\alpha}(|z|) d \nu(z) \\
& =\frac{2 n}{c_{\alpha 1}}\left\{\|f\|_{A_{\varphi}\left(v_{\alpha}\right)}-\varphi(\log |f(0)|)\right\}<\infty,
\end{aligned}
$$


where $c_{\alpha 1}=1 / 2$ if $\alpha=-1$.

(b) implies (a). By Lemma 3.10,

(4) $\|f\|_{A_{\varphi}\left(v_{\alpha}\right)}-\varphi(\log |f(0)|)=\frac{1}{2 n} \int_{B} f_{\varphi}^{\sharp}(z)|z|^{-2(n-1)} K_{\alpha}(|z|) d \nu(z)$

$$
=\frac{1}{2 n}\left(\int_{\frac{1}{2} B}+\int_{B \backslash \frac{1}{2} \bar{B}}\right) f_{\varphi}^{\sharp}(z)|z|^{-2(n-1)} K_{\alpha}(|z|) d \nu(z) .
$$

By Lemma 3.14,

$$
\int_{\frac{1}{2} B} f_{\varphi}^{\sharp}(z)|z|^{-2(n-1)} K_{\alpha}(|z|) d \nu(z)<\infty .
$$

By Lemma 3.1 and (b),

$$
\text { (6) } \begin{aligned}
\int_{B \backslash \frac{1}{2} \bar{B}} f_{\varphi}^{\prime}(z)|z|^{-2(n-1)} K_{\alpha}(|z|) d \nu(z) & \leq 2^{2(n-1)} c_{\alpha 2} \int_{B \backslash \frac{1}{2} \bar{B}} f_{\varphi}^{\sharp}(z)\left(1-|z|^{2}\right)^{\alpha+2} d v(z) \\
& \leq 2^{2(n-1)} c_{\alpha 2} \int_{B} f_{\varphi}^{\sharp}(z)\left(1-|z|^{2}\right)^{\alpha+2} d \nu(z)<\infty,
\end{aligned}
$$

where $c_{\alpha 2}=1$ if $\alpha=-1$. By (4), (5) and (6), we have $\|f\|_{A_{\varphi}\left(v_{\alpha}\right)}<\infty$. This shows that $f \in A_{\varphi}\left(v_{\alpha}\right)$.

(c) implies (d). This is trivial.

(d) implies (b). Fix a number $r \in\left(r_{\beta}, 1\right)$. Using Lemma 3.1 and Lemma 3.12, we have

$$
\begin{aligned}
& \int_{B} f_{\varphi}^{\sharp}(z)\left(1-|z|^{2}\right)^{\alpha+2} d \nu(z) \\
&=\left(\int_{r B}+\int_{B \backslash r \bar{B}}\right) f_{\varphi}^{\sharp}(z)\left(1-|z|^{2}\right)^{\alpha+2} d \nu(z) \\
& \leq \frac{1}{c_{\alpha 1}} \int_{r B} f_{\varphi}^{\sharp}(z)|z|^{-2(n-1)} K_{\alpha}(|z|) d \nu(z) \\
& \quad+\int_{B \backslash r \bar{B}} f_{\varphi}^{\sharp}(z)\left(1-|z|^{2}\right)^{\alpha+2}|z|^{-2(n-1)} d \nu(z) \\
& \leq \frac{1}{c_{\alpha 1}} \int_{r B} f_{\varphi}^{\sharp}(z)|z|^{-2(n-1)} K_{\alpha}(|z|) d \nu(z)+\frac{1}{\inf _{r<r<1} G_{\beta}(t)} \mathscr{L}_{\varphi}(f, \alpha, \beta) .
\end{aligned}
$$

Since $r_{\beta}<r<1$, by Lemma 3.11,

$$
0<\frac{1}{\inf _{r<t<1} G_{\beta}(t)}<\infty
$$

By (7), Lemma 3.14, (8) and (d), we obtain (b). 
(b) implies (c). Fix any $\beta \in(1, \infty)$. By Lemma 3.12,

$$
\mathscr{L}_{\varphi}(f, \alpha, \beta) \leq\left\{\sup _{0 \leq t<1} G_{\beta}(t)\right\} \int_{B \backslash r_{\beta} \bar{B}} f_{\varphi}^{\sharp}(z)\left(1-|z|^{2}\right)^{\alpha+2}|z|^{-2(n-1)} d v(z) .
$$

By Lemma 3.11,0< $\gamma_{\beta} \equiv \sup _{0 \leq t<1} G_{\beta}(t)<\infty$. Hence we have

$$
\begin{aligned}
\mathscr{L}_{\varphi}(f, \alpha, \beta) & \leq \gamma_{\beta} r_{\beta}^{-2(n-1)} \int_{B \backslash r_{\beta} \bar{B}} f_{\varphi}^{\sharp}(z)\left(1-|z|^{2}\right)^{\alpha+2} d \nu(z) \\
& \leq \gamma_{\beta} r_{\beta}^{-2(n-1)} \int_{B} f_{\varphi}^{\sharp}(z)\left(1-|z|^{2}\right)^{\alpha+2} d \nu(z)<\infty .
\end{aligned}
$$

This completes the proof.

\section{Acknowledgement}

The authors would like to thank the referee for valuable comments and suggestions.

\section{References}

[1] F. Beatrous and J. Burbea, 'Characterizations of spaces of holomorphic functions in the ball', Kodai Math. J. 8 (1985), 36-51.

[2] C. Ouyang and J. Riihentaus, 'A characterization of Hardy-Orlicz spaces on $\mathrm{C}^{n}$, Math. Scand. 80 (1997), 25-40.

[3] W. Rudin, Function theory in the unit ball of $\mathbf{C}^{n}$ (Springer, Berlin, 1980).

[4] M. Stoll, 'Harmonic majorants for plurisubharmonic functions on bounded symmetric domains with applications to the spaces $H_{\Phi}$ and $N_{*}^{\prime}$ ', J. Reine Angew. Math. 282 (1976), 80-87.

[5] —_. 'A characterization of Hardy-Orlicz spaces on planar domains', Proc. Amer. Math. Soc. 117 (1993), 1031-1038.

[6] _ - 'A characterization of Hardy spaces on the unit ball of $C^{n}$ ', J. London Math. Soc. 48 (1993), 126-136.

[7] - Invariant potential theory in the unit ball of $\mathbf{C}^{n}$ (Cambridge Univ. Press, 1994).

[8] S. Yamashita, 'Criteria for functions to be of Hardy class $H^{p}$, Proc. Amer. Math. Soc. 75 (1979), 69-72.

\section{Department of Mathematical Sciences}

Faculty of Science

Shinshu University

390-8621 Matsumoto

Japan

e-mail: matsugu@math.shinshu-u.ac.jp 
